\title{
St. Gallen 2007: \\ Breast Cancer Treatment Consensus Report
}

\author{
Nadia Harbeck $^{\mathrm{a}}$ Raimund Jakesz ${ }^{\mathrm{b}}$ \\ ${ }^{a}$ Frauenklinik der Technischen Universität München, Germany \\ ${ }^{b}$ Klinische Abteilung für Allgemeinchirurgie, Medizinische Universität Wien, Austria
}

\section{Key Words}

Breast cancer - Endocrine treatment - Chemotherapy . Antibody therapy - Targeted therapy

\section{Summary}

The focus of this year's St. Gallen Consensus Conference was on providing substantial and largely evidence-based recommendations for the treatment of early breast cancer, indicating a broad spectrum of acceptable clinical practice. This report summarises the results of the 2007 international panel voting procedures with regard to endocrine treatment, chemotherapy and antibody therapy.

\section{Introduction}

The motto of this year's St. Gallen Consensus Conference, taking place March 14-17, was 'Care despite Controversy', with a particular emphasis on treatment recommendations rather than guidelines. The panel consisted of selected breast cancer experts predominantly from Europe and the USA (fig. 1). William Wood (Atlanta, USA), who chaired the Saturday morning consensus session, pointed out that the consensus is aimed at i) updating information for improved treatment choices, and ii) improving the understanding of information from trials that are typically aimed at testing therapies and less focussed on efficacy issues or the benefit for an individual patient. The St. Gallen conference 2007 also looked closely at targeted therapies and their broadest possible application. The general treatment recommendations, given as usual on Satur-

\section{Schlüsselwörter}

Mammakarzinom - Endokrine Therapie - Chemotherapie · Antikörpertherapie - Targeted Therapy

\section{Zusammenfassung}

Der Schwerpunkt der diesjährigen St. Gallen Konsensuskonferenz lag auf der Erstellung substantieller und im Wesentlichen evidenzbasierter Empfehlungen für die Behandlung des Mammakarzinoms im Frühstadium. Ein breites Spektrum an akzeptabler klinischer Praxis wurde vorgegeben. Der vorliegende Bericht fasst die Ergebnisse der Abstimmung des internationalen Panels im Hinblick auf endokrine Therapie, Chemotherapie und Antikörpertherapie zusammen.

day morning (March 17), did not differ essentially from the updated St. Gallen 2005 recommendations (fig. 2) [1, 2].

In order to obtain clinically relevant statements from the experts, questions were asked with 3 answer options (yes/no/abstain). The answers were summarised in percentages. Since sometimes several questions addressed the same issue, the percentages reported here do not necessarily add up to $100 \%$. Rather, the questions tended to be general, and the answers indicated a corridor of accepted clinical practice according to the international experts. The answers thus did not provide specific recommendations for individual patients nor a clinically controversial scenario.

In general, it is crucial to understand the different types of evidence available to support the answers given by the panel. Some topics were addressed by meta-analyses into which many thousands of patients had been included. Other evidence

\begin{tabular}{ll}
\hline KARGER & ๑ 2007 S. Karger GmbH, Freiburg \\
Fax +49 7614520714 & Accessible online at: \\
$\begin{array}{l}\text { E-mail Information@Karger.de } \\
\text { www.karger.com }\end{array}$ & www.karger.com/brc
\end{tabular}




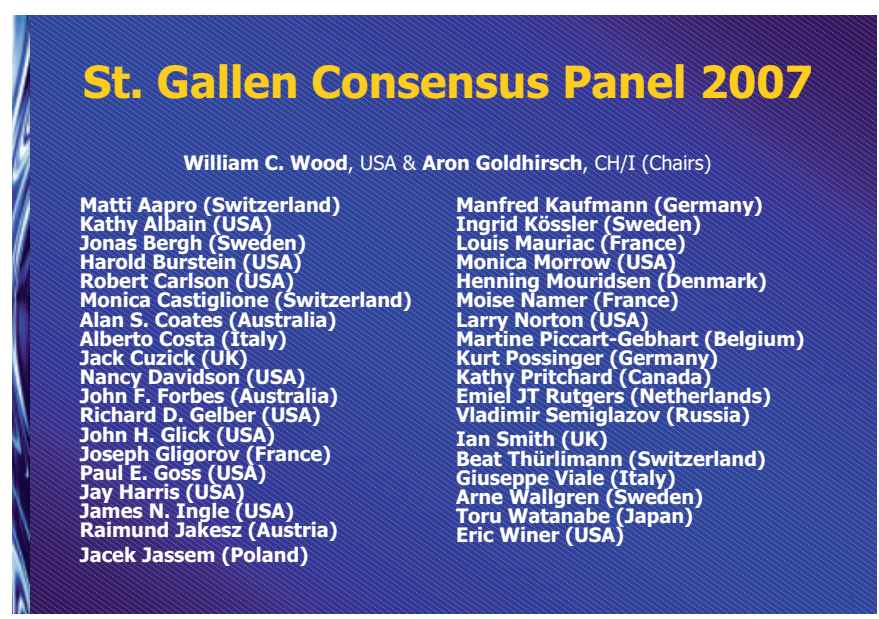

Fig. 1. St. Gallen consensus panel 2007.

comes from several randomised trials, at times with different and conflicting results, and some outcomes emerge from analysis of experience. But there are also topics not substantiated by trial results but rather by deductive conclusions that can be drawn from other, similar clinical situations. Finally, there are also clinical situations in which prospective randomised trials will never be performed, but which still represent important topics to be addressed together with the patients.

\section{Endocrine Treatment}

For many years, endocrine therapies have been playing an important role in the adjuvant systemic treatment of patients with breast cancer. Endocrine treatment was the first targeted therapy based on an understanding that its treatment effect is mediated by the oestrogen receptors (ER) and progesterone receptors $(\mathrm{PgR})$ in breast cancer cells.

In 2005, the panel divided tumour endocrine responsiveness into a highly responsive and an uncertain responsive category, and basically reaffirmed these categories in 2007, now referring to high and partial endocrine responsiveness, respectively. Endocrine responsiveness is characterised by a higher expression of both ER and PgR in the majority of tumour cells. Partial endocrine responsiveness implies a lower expression of ER and/or PgR, although no absolute threshold can so far be defined. Endocrine responsiveness should be seen in the context of risk categories, taking into account not only the expression of steroid hormone receptors, but also the status of extensive peritumoural vascular invasion (PVI), human epidermal growth factor receptor 2 (HER2) overexpression or gene amplification, and patient age. The definition of risk categories low, intermediate and high risk - has in principle remained unchanged since the 2005 statement (fig. 3).

Several speakers clearly pointed out that it is essential to consider breast cancer as a highly heterogeneous disease, based on the magnitude of expression not only of the receptor status,

\begin{tabular}{|c|c|c|c|}
\hline \multicolumn{4}{|c|}{$\begin{array}{l}\text { St. Gallen Consensus } \\
2005-2007\end{array}$} \\
\hline Risk group & $\begin{array}{l}\text { Endocrine } \\
\text { responsive }\end{array}$ & $\begin{array}{l}\text { Doubtful } \\
\text { endocrine } \\
\text { responsive }\end{array}$ & $\begin{array}{l}\text { Endocrine } \\
\text { non } \\
\text { responsive }\end{array}$ \\
\hline $\begin{array}{l}\text { Low } \\
\text { Risiko }\end{array}$ & ET & ET & \\
\hline $\begin{array}{l}\text { Intermediate } \\
\text { risk } \\
\text { HER2 + }\end{array}$ & $\begin{array}{l}\text { ET alone or } \\
\text { CT } \rightarrow \text { ET } \\
\text { Trastuzumab }\end{array}$ & $\begin{array}{l}\text { CT } \rightarrow \text { ET } \\
\text { Trastuzumab }\end{array}$ & $\begin{array}{l}\text { CT } \\
\text { Trastuzumab }\end{array}$ \\
\hline $\begin{array}{l}\text { High } \\
\text { Risk } \\
\text { HER2 + }\end{array}$ & $\begin{array}{l}\text { CT } \rightarrow \text { ET } \\
\text { Trastuzumab }\end{array}$ & $\begin{array}{l}\mathrm{CT} \rightarrow \mathrm{ET} \\
\text { Trastuzumab }\end{array}$ & $\begin{array}{l}\text { CT } \\
\text { Trastuzumal }\end{array}$ \\
\hline =endocrine therapy: C & notherapy & Modified after Goldhirsc & et al, Ann Oncol 2006 \\
\hline
\end{tabular}

Fig. 2. St. Gallen consensus 2005-2007.

but also of HER2. There are also other markers which in various ways are important for the prediction of response to a treatment. Microarray differentiation defines at least 6 different subtypes of breast cancer - luminal types A, B and C, normal breast-like, HER2-positive, and basal-like phenotypes [3]. During the meeting, an update of state-of-the-art adjuvant endocrine treatment for premenopausal patients was given by Nancy Davidson [4]. Since the last overview, a meta-analysis of trials including luteinising hormone-releasing hormone (LHRH) analogues has been presented by Jack Cuzick at the 2006 San Antonio Breast Cancer Symposium (SABCS) [5] and has recently been published [6]. Over the 1987-2001 period, close to 12,000 ER- and/or PgR-positive patients were recruited in different trials, with a median follow-up of 6.8 years. The major findings were that LHRH analogues showed a nonsignificant reduction in recurrence rate $(\mathrm{p}=0.08)$, with a $28.4 \%$ relative reduction and smaller but significant improvements in recurrence-free survival (RFS) $(p=0.02)$ and overall survival $(\mathrm{OS})(\mathrm{p}=0.03)$ when LHRH analogues were used together with tamoxifen, chemotherapy or both.

With respect to endocrine treatment in premenopausal patients, more than $80 \%$ of the panel opted for tamoxifen and for ovarian function suppression (OFS) + tamoxifen, especially so in women at low risk and those planning to become pregnant. The patients should be treated with OFS + tamoxifen for 5 years, especially when they are node-positive and/or HER2-positive (66\% approval). Administration of an LHRH analogue is the appropriate way to suppress ovarian function. Surgical ovariectomy is also an appropriate option (76\%), in contrast to ovarian irradiation (fig. 4). The panel opted for sequential treatment when chemotherapy and OFS are to be delivered $(82 \%)$. The combination of aromatase inhibitors (AIs) and OFS is only regarded as appropriate if patients have a tamoxifen contraindication (68\%). Supportive care for premenopausal patients who receive endocrine treatment should be given with respect to menopausal symptoms (55\%) and impaired bone mineral density (62\% approval). 


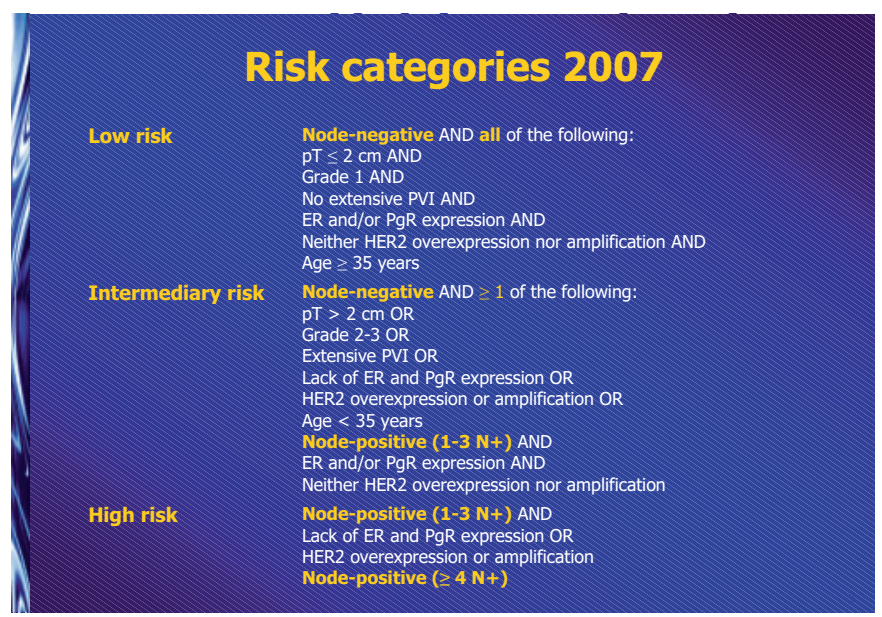

Fig. 3. Risk categories 2007.

The key lecture regarding endocrine treatment of postmenopausal patients was given by Paul Goss [7], dealing mainly with AIs. AIs can be used in the adjuvant stage at 3 different postoperative time points: i) as primary treatment directly subsequent to surgery, ii) after initial treatment with tamoxifen for 2-3 years, or iii) as extended treatment after 5 years of primary tamoxifen treatment. Three drugs are currently available: anastrozole, letrozole and exemestane. In terms of primary treatment, anastrozole and letrozole have demonstrated a significant improvement in 4-year disease-free survival (DFS) with hazard ratios (HRs) of 0.83 for the Anastrozole, Tamoxifen, Alone or in Combination (ATAC) trial and 0.81 for the Breast International Group (BIG) 01.98 study, however, with no benefits in OS. Retrospective analysis of the 2 trials with primary AI treatment measuring ER, PgR and HER2 status in a singlereference lab has shown no predictive value attached to any of these factors in terms of increased AI efficacy. In terms of an early switch of treatment, exemestane and anastrozole have shown to improve 4-year DFS to a greater extent, with HRs of 0.73 for the International Exemestane Study (IES) and 0.60 in a recent analysis of Austrian Breast and Colorectal Cancer Study Group (ABCSG) Trial 8 and the German ArimidexNolvadex (ARNO) study [8]. A meta-analysis of the 3 switching studies with anastrozole has recently shown an improvement in OS (HR 0.79) [9].

An investigation of extended AI treatment - the MA.17 trial demonstrated letrozole to result in significantly improved DFS rates with a HR of 0.58 [10]. Likewise, ABCSG Trial 6a, exploring extended adjuvant treatment with anastrozole vs. surveillance, identified a significantly reduced risk of local, contralateral and distant metastatic recurrence in patients receiving active AI therapy (HR 0.62) [11].

As to the questions addressed to the panel with respect to endocrine treatment in postmenopausal patients, the experts voted overwhelmingly that tamoxifen is still an option in this indication ( $76 \%$ approval). More than $80 \%$ of the panel mem-

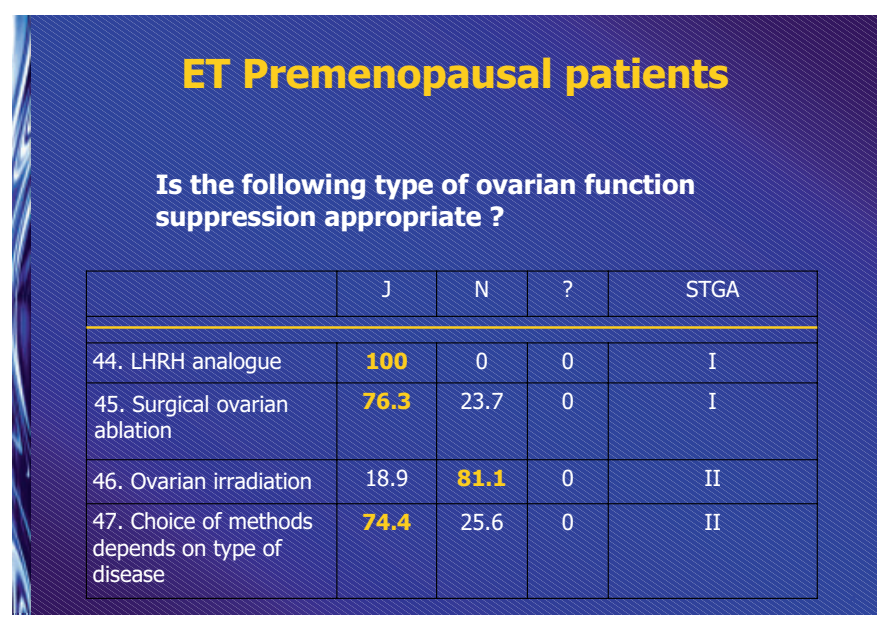

Fig. 4. Endocrine therapy (ET) in premenopausal patients.

bers voted for a switch to an AI after 2-3 years of tamoxifen. $70 \%$ were in favour of applying an $\mathrm{AI}$ after primary tamoxifen treatment for 5 years. A substantial number of panellists $(60 \%)$ voted for primary AI treatment in high-risk patients, and especially in those who are HER2-positive, although no data supporting that strategy are presently available. According to the majority (>90\%), extended endocrine treatment with AIs after 5 years of tamoxifen should be restricted to node-positive patients. Therefore, treatment should be given for a period of 5 to 10 years - there was no majority to endorse the idea of life-long endocrine treatment.

The option of checking ovarian function in order to establish the menopausal status as a prerequisite for an $\mathrm{AI}$ indication left the panel uncertain. Routine bisphosphonate treatment was not supported by the panellists, quite contrary to $100 \%$ of panel members emphasizing the value of physical exercise (fig. 5).

In the case of postmenopausal patients with node-negative disease, a positive hormone receptor, and HER2 positivity, the panel voted in favour of giving these women a combination of endocrine and trastuzumab treatment without chemotherapy. In patients with uncertain endocrine sensitivity, and thus the need for chemotherapy and endocrine treatment, more than $80 \%$ endorsed sequential treatment.

\section{Chemotherapy}

As pointed out above, due to the applied question-and-answer process, St. Gallen 2007 was not intended to give specific chemotherapy recommendations, but rather proposed a number of acceptable regimens and outlined risk categories for their use. Questions regarding chemotherapy addressed the following issues: i) type of treatment, ii) type of regimen, iii) supportive therapies, and iv) indications for chemotherapy ('method of choice'). 


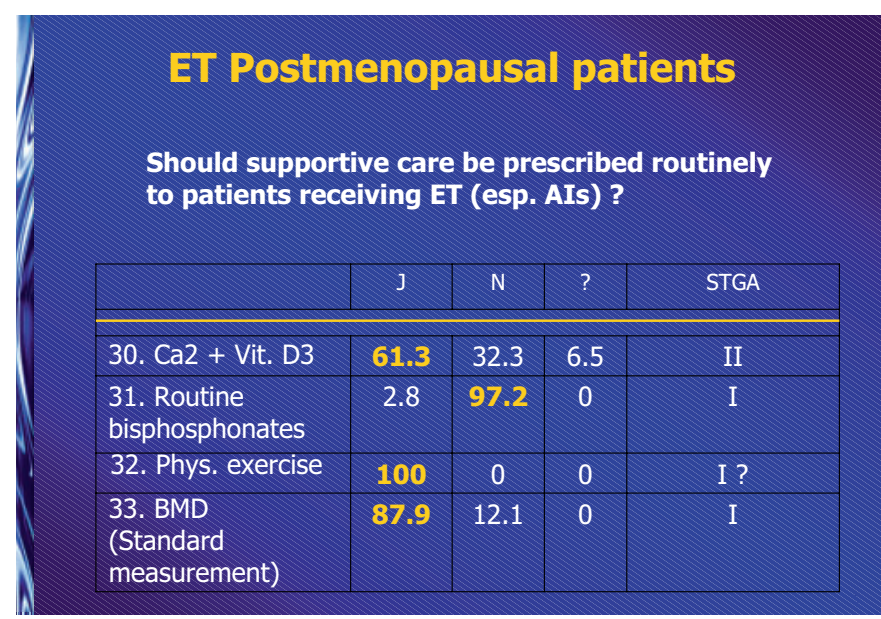

Fig. 5. Endocrine therapy (ET) in postmenopausal patients.

With regard to the type of treatment, the panel did not favour a different choice of chemotherapy for HER2-positive versus HER2-negative tumours ( $41 \%$ same chemotherapy, $38 \%$ not the same, $22 \%$ abstention). The vast majority ( $85 \%$ ) opted for anthracycline-containing chemotherapy in HER2-positive patients, and only $15 \%$ would avoid anthracyclines in this population. A special emphasis throughout the St. Gallen Conference and the consensus session was placed on biological subgroups as defined by Sorlie et al. [12]. In triple-negative (HER2-, ER- and PgR-negative) patients, the panel did not endorse platinum-containing chemotherapy (only 15\% approval) in the adjuvant setting, yet alkylating regimens were supported by the majority ( $82 \%$ ) in this indication. $59 \%$ (vs. $38 \%$ ) thought that the type of chemotherapy regimen should be chosen regardless of nodal status. While the majority endorsed anthracycline therapy for all patients (73\%), only $40 \%$ supported taxanes for all (57\% disapproval). There seemed to be a general agreement (64\%) that ideal adjuvant chemotherapy should consist of $6-8$ cycles. Only $44 \%$ considered $4 \times$ doxorubicine/cyclophosphamide (AC) to still be a viable regimen, and even less (23\%) still accepted 6x cyclophosphamide/ methotrexate/fluorouracil (CMF).

Different regimens were discussed, and the choices reflected the international composition of the panel. Complete agreement existed on the subject of high-dose chemotherapy supported by peripheral blood stem cell support - it was rejected as a standard regimen (100\% disapproval). In general, there was agreement on the anthracycline-type regimens: the cyclophosphamide/doxorubicin/fluorouracil (CAF) or Canadian cyclophosphamide/epirubicin/fluorouracil $\left(\mathrm{CE}_{120} \mathrm{~F}\right)$ types $(63 \%)$ and FAC- or FEC-type regimens $(61 \%)$ found broad acceptation within the panel. In contrast, each of the various taxane-containing regimens received a lower approval rate: $37 \%$ for doxorubicin/cyclophosphamide/paclitaxel (AC-T) and $30 \%$ for docetaxel/doxorubicin/cyclophosphamide (TAC). In HER2-negative disease, FEC-docetaxel (standard regimen:
$25 \%$ ) and $4 \times$ docetaxel/cyclophosphamide (TC) (standard regimen: $32 \%$ ) were also discussed as taxane-containing therapy options. Precisely half of the panel accepted dose-dense EC or AC-paclitaxel regimens as valid treatment options. While haematopoietic growth factors were generally accepted as standard supportive therapy ( $47 \%$ approval), other supportive measures such as cardioprotectors were seen less favourably ( $6 \%$ approval).

Regarding the indication for adjuvant chemotherapy, $94 \%$ accepted it as standard treatment in patients with $>4$ involved lymph nodes, $83 \%$ in less involved lymph nodes, and even $88 \%$ in high-risk node-negative patients, at least in the presence of uncertain endocrine responsiveness. The panel, however, appeared very cautious in making use of novel risk assessment tools, such as adjuvant!online, Oncotype DX or Mammaprint (with 47, 31 and 5\% approvals, respectively), for indicating adjuvant chemotherapy in uncertain endocrine-responsive breast cancer - even assuming these tools were readily available.

Preoperative ('neoadjuvant') chemotherapy was not discussed in depth at the Saturday morning session, and the experts were only asked about specific indications. The majority accepted the use of trastuzumab (in HER2-positive disease) during the neoadjuvant treatment phase ( $82 \%$ approval), whereas only $31 \%$ thought that a taxane should always be included. Better cosmesis was an indication which most experts ( $72 \%)$ considered valid, whereas endocrine non-responsive disease or testing chemoresponsiveness in tumours $<5 \mathrm{~cm}$ were not considered sufficient for initiating primary systemic therapy ( 42 and $35 \%$, respectively).

\section{Antibody Therapy}

Since 2005, the presence of HER2 on the cell surface as a target for trastuzumab has emerged as a valuable target in addition to the previously recognised hormone receptors as targets for endocrine therapies [13-15]. As already stated in the 2006 interim update of the 2005 St. Gallen recommendations [2], HER2 status was accepted as an obligatory parameter for risk group stratification. Likewise, the experts this year accepted trastuzumab therapy (in HER2-positive disease) as adjuvant standard therapy. $92 \%$ considered immunohistochemistry as the standard method for HER2 determination, with only a minority (16\%) demanding fluorescence in situ hybridisation (FISH) testing in all cases.

In node-negative disease, $58 \%$ accepted trastuzumab for endocrine-responsive tumours, yet only $33 \%$ thought that trastuzumab should be given if such tumours are smaller than $1 \mathrm{~cm}$. In contrast, $56 \%$ accepted trastuzumab therapy in such small node-negative tumours if they were endocrine non-responsive. One year of trastuzumab therapy was confirmed as the standard by an overwhelming majority $(92 \%)$, whereas only $14 \%$ considered the short Finland Herceptin (FinHER) 
schedule as a valid option. $58 \%$ of the experts considered trastuzumab therapy as a valid adjuvant option if administered exclusively with endocrine therapy and no chemotherapy (38\% disapproval).

The experts had no distinct preference regarding the optimal chemotherapy regimen to be given together with trastuzumab therapy, or the optimal timing of trastuzumab in relation to chemotherapy. $38 \%$ preferred the sequential Herceptin Adjuvant (HERA) trial model compared to $41 \%$ favouring the concomitant approach and $22 \%$ for whom both options were equally acceptable. About half of the experts (51\%) considered docetaxel/carboplatin/trastuzumab (TCH) to be an important option in HER2-positive disease. Only 30\% deemed age an important consideration for indicating adjuvant trastuzumab, whereas $74 \%$ stated that they would avoid trastuzumab in patients with low left ventricular ejection fraction.

\section{Conclusion}

St. Gallen 2007 produced a clear consensus based on a few general principles - focusing on target identification - within which a wide range of acceptable options were identified. As it was to be expected in view of the heterogeneous international consensus panel, recommendations regarding specific chemotherapy regimens were not given this year.

Summarising the data on endocrine treatment, tamoxifen is still considered a viable option. In postmenopausal patients, there is a clear agreement for AI therapy which should be given using a risk-adapted perspective, although predictive markers are currently not available. A majority favoured switching from tamoxifen to AIs. In the premenopausal setting, the role of LHRH analogues has been substantiated. Chemo-endocrine treatment in combination with LHRH analogues (for ovarian protection) may be offered to patients planning subsequent pregnancy. Otherwise, sequential chemoendocrine therapy is preferable. In postmenopausal patients with node-negative disease, it is considered an option to give endocrine treatment together with trastuzumab even without administering chemotherapy.

In general, 6-8 cycles of chemotherapy were favoured, and an anthracycline-containing regimen seemed to be accepted as a backbone of adjuvant chemotherapy. Regional differences based on national trial histories were most obvious when it came to the most preferable anthracycline-taxane combinations. Regarding HER2-positive disease, adjuvant and neoadjuvant trastuzumab therapy were considered standard. Yet, no consensus was reached with regard to the preferred regimen in the adjuvant setting, and trastuzumab therapy was accepted either sequentially to or concomitant to adjuvant chemotherapy.

In conclusion, the recommendations of the St. Gallen Consensus Panel provide a minimal standard for up-to-date breast cancer treatment which are based on expert opinions as well as published trial data. National guidelines may well differ from these recommendations due to the resources available in individual countries and different interpretations of available evidence. The annually updated Arbeitsgemeinschaft Gynäkologische Onkologie (AGO) guidelines [16] that systematically review the recent peer-reviewed publications as well as American Society of Clinical Oncology (ASCO) and SABCS abstracts do provide a practical framework for evidence-based breast cancer therapy.

\section{References}

1 Goldhirsch A, Glick JH, Gelber RD, et al.: Meeting highlights: international expert consensus on the primary therapy of early breast cancer 2005 . Ann Oncol 2005;16:1569-1583.

$>2$ Goldhirsch A, Coates AS, Gelber RD, et al.: First select the target: better choice of adjuvant treatments for breast cancer patients. Ann Oncol 2006; 17:1772-1776.

3 Fan C, Oh DS, Wessels L, et al.: Concordance among gene expression-predictors for breast cancer. N Engl J Med 2006;355:560-569.

4 Davidson N: Ovarian function suppression in the adjuvant program. Primary Therapy of Early Breast Cancer: 10th International St. Gallen Conference, 2007, oral presentation.

5 Cuzick J: The impact of LHRH agonists on breast cancer recurrence and mortality. 29th San Antonio Breast Cancer Symposium, 2006, oral presentation.

6 Cuzick J, Ambroisine L, Davidson N: Use of luteinising-hormone-releasing hormone agonists as adjuvant treatment in premenopausal patients with hormone-receptor-positive breast cancer: A meta-analysis of individual patient data from randomised adjuvant trials. Lancet 2007;369:1711-1723.
7 Goss P: Application of aromatase inhibitors in endocrine responsive breast cancer. Primary Therapy of Early Breast Cancer: 10th International St. Gallen Conference, 2007, oral presentation.

8 Jakesz R, Jonat W, Gnant M, et al.: Switching of postmenopausal women with endocrine-responsive early breast cancer to anastrozole after 2 years' adjuvant tamoxifen: combined results of ABCSG trial 8 and ARNO 95 trial. Lancet 2005;366:455-462.

9 Jonat W, Gnant M, Boccardo F, et al.: Effectiveness of switching from adjuvant tamoxifen to anastrozole in postmenopausal women with hormone-sensitive early-stage breast cancer: a meta-analysis. Lancet Oncol 2006;7:991-996.

10 Goss PE, Ingle JI, Martino S, et al.: Randomized trial of letrozole following tamoxifen as extended adjuvant therapy in receptor-positive breast cancer: updated findings from NCIC CTG MA.17. J Nat Cancer Inst 2005;97:1262-1271.

11 Jakesz R, Greil R, Gnant M, et al.: Extended adjuvant therapy with anastrozole: results from Austrian Breast and Colorectal Cancer Study Group Trial 6A. J Nat Cancer Inst, submitted.
2 Sorlie T, Perou CM, Tibshirani R, et al.: Gene expression patterns of breast carcinomas distinguish tumor subclasses with clinical implications. Proc Natl Acad Sci U S A 2001;98:10869-10874.

13 Piccart-Gebhart MJ, Procter M, Leyland-Jones B, et al.: Trastuzumab after adjuvant chemotherapy in HER2-positive breast cancer. N Engl J Med 2005; 353:1659-1672.

14 Romond EH, Perez EA, Bryant J, et al.: Trastuzumab plus adjuvant chemotherapy for operable HER2-positive breast cancer. N Engl J Med 2005; 353:1673-1684.

15 Slamon D: BCIRG 006 II interim analysis. San Antonio Breast Cancer Symposium, 2006, oral presentation.

16 AGO Kommission Mamma: Diagnosis and treatment of patients with primary and metastatic breast cancer: guidelines of the AGO Breast Commission. www.ago-online.org. 